\title{
Modeling incision profile in AWJM of Titanium alloys Ti6AI4V
}

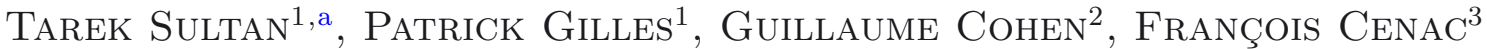 \\ AND WALTER RUBio ${ }^{2}$ \\ 1 Institut Clément Ader/INSA, Toulouse 135 Avenue de Rangueil 31077, Toulouse Cedex 4, France \\ 2 Institut Clément Ader/Université Toulouse III, Paul Sabatier, 118 route de Narbonne 31062, Toulouse Cedex 9, France \\ 3 JEDO Technologies, Rue du Chêne Vert, BP 78204, 31682 Labège, France
}

Received 29 October 2014, Accepted 23 October 2015

\begin{abstract}
Abrasive water jet milling (AWJM) is a new way to perform controlled depth milling especially for hard materials, but it's not yet enough reliable because of large variety of process parameters and complex footprint geometries that are not well mastered. In order to master the milling device in AWJM, a deep study on the footprint of a single path of the cutting head should first be considered. The flow of the AWJ and the distribution of abrasive particles coming out of the jet are related to the profile measured on the footprint. In this study, experiments were made on titanium alloys specimen to compare several theoretical models to the measured profile of the footprint. This study establishes new models to fit the incision profile taking in consideration the behavior of the abrasive particles impacting the workpiece.
\end{abstract}

Key words: Abrasive water jet milling / incision / titanium alloy / process parameters / Gaussian model

\section{Nomenclature}

\begin{tabular}{|ll|}
\hline$a$ & Maximum depth parameter $(\mathrm{mm})$ \\
$b$ & Width parameter (mm) \\
$b_{0.5}$ & Width at half of maximum depth $(\mathrm{mm})$ \\
$b_{1}$ & Width parameter of first Gaussian part $(\mathrm{mm})$ \\
$b_{2}$ & Width parameter of second Gaussian part $(\mathrm{mm})$ \\
$D a$ & Abrasive flow rate $\left(\mathrm{L} \cdot \mathrm{min}^{-1}\right)$ \\
$D c$ & Ocusing tube diameter $(\mathrm{mm})$ \\
$f$ & Traverse speed $\left(\mathrm{mm}^{-1} \mathrm{~min}^{-1}\right)$ \\
$h$ & Incision's depth $(\mathrm{mm})$ \\
$K$ & Width parameter of Pearson equation $(\mathrm{mm})$ \\
$M$ & Width parameter of Pearson equation $(\mathrm{mm})$ \\
$P$ & Pressure (bar) \\
$S O D$ & Standoff distance $\left(\mathrm{mm}^{2}\right)$ \\
$S S E$ & Sum square error $\left(\mathrm{mm}^{2}\right)$ \\
\hline
\end{tabular}

\section{Introduction}

Abrasive water jet (AWJ) acquired a great technological progress in the last decades. Its popularity has

\footnotetext{
a Corresponding author:

tarek.sultan@insa-toulouse.fr
}

changed considerably thanks to its high pressure joined to the flow of abrasive particles allowing the cut of any type of material. AWJ is a non-conventional machining process in which a mixture of abrasive particles and water at very high pressure is converted into a high velocity jet to cut various materials that can range from brittle to ductile materials. The water jet cutting is considered as a very efficient manufacturing process involving small amounts of heat transferred to the workpiece. Thereby, metal parts made by AWJ process do not change the crystal structure usually caused by the heat generated with other methods of material removal such as laser cutting, EDM (Electrical Discharge Machining), milling machining... The AWJ has become an emerging technology with versatile features.

Controlled depth milling appeared in the early 1990s and is intended for the production of pockets. Until now this process has not yet been mastered, especially for hard materials. In order to comprehend the milling of pockets in AWJ, milling incisions (representing a single path of the cutting head on the workpiece) should firstly be studied. Several works were established to investigate this technology, Cenac studied controlled depth milling on Composites and aluminum alloys [1,2], and Flower worked on Titanium alloys [3]. In their studies masks were used to avoid irregularities in the bottom of the milled pocket. In the case of maskless pocket milling in AWJ, a precise 


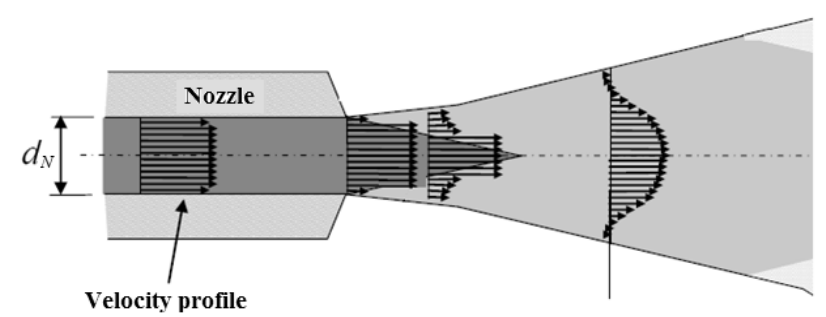

Fig. 1. Water particles velocity profile according to Cenac [1].

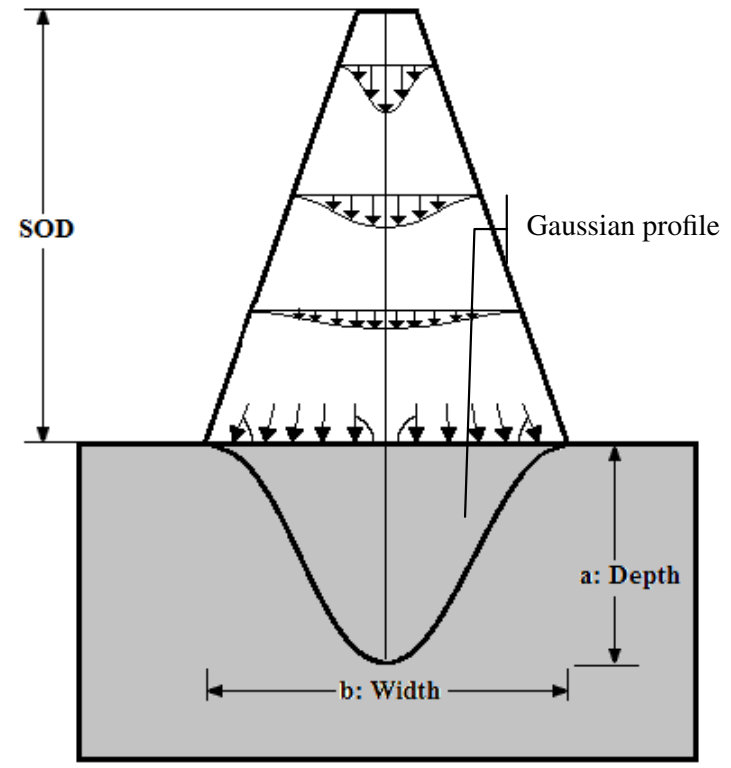

Fig. 2. Velocity jet profile according to Srinivasu [5].

study on the impinging water jet is required to understand the mechanisms of abrasive and water flow machining and their impact on the workpiece.

Cenac showed in his studies [1] that the velocity profile of water particles going out of the cutting head (Fig. 1) brings to mind a Gaussian profile as described in the literature [4]. Srinivasu and Axinte [5] also associated the bell's profile obtained on the material to be milled after one path of the jet to the fluid behavior of water particles outgoing the cutting head (Fig. 2). It is to be noted that the Gaussian shape is found on any kind of material.

This assumption is not justified in all cases since the fluidic behavior of these particles can differ by varying the parameters of the machine. Zuckerman et al. [6] analyzed the behavior of the jet at high velocity (Fig. 3). They showed that the abrasive particles coming out through the jet and gaining kinetic energy due to waterjet won't have a regular influence on the workpiece. Initially, with high pressure, the material to be milled is ploughed by the fine abrasives outgoing along the jet axis which come in contact with the workpiece as the rub over the surface [7-9]. It is to be noted that there is another mechanism of abrasive flow during AWJ milling. In addition of ploughing mechanism, some particles on the sideways of

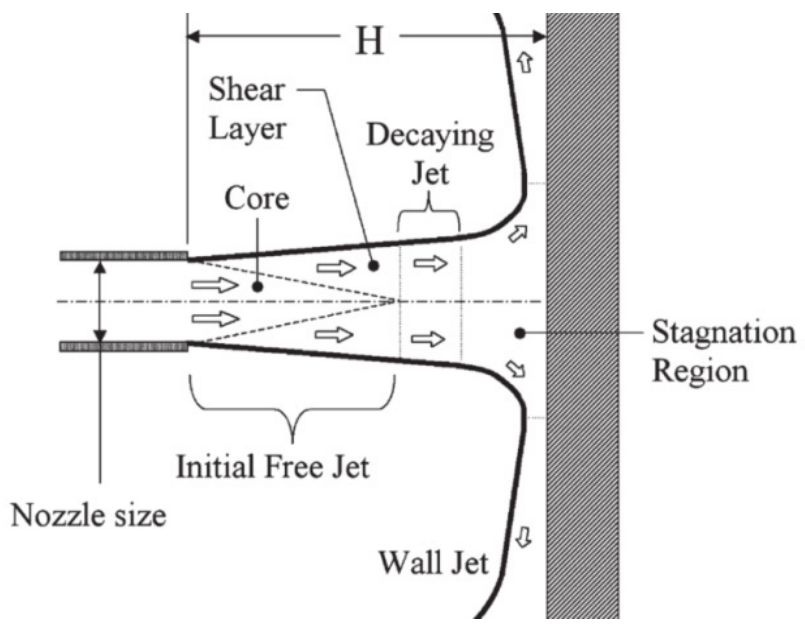

Fig. 3. The flow of impinging water jet according to Zuckerman and Lior [6].

the water flow erode the surface of the workpiece with micro cutting action [10-12]. Thus, during this mechanism, the abrasive particles physically remove the material by eroding the surface producing micro incisions. This mechanism is seen in the material removal processes such as grinding.

\subsection{Incision profile analysis}

While milling an incision in AWJ, abrasive particles in the lateral direction of the jet tend to erode the surface. The aim of this work is to analyze the shape of the kerf resulting from a single straight passage of the cutting head performing what is called an incision in AWJM. Any technological machining method used for the generation of surfaces leaves some technical irregularities which have fundamental influence on functional surfaces. In fact, the first step is to understand the shape of incisions before being concerned about milling pockets in AWJ. Therefore, a study of the correlation between the experimental incisions milled by AWJ machining and a consistent theoretical model is required. The theoretical models are based on mathematical equations defining the profile generated by a single path of the jet on the material (Fig. 4), here titanium alloys Ti6AlV are expressed.

Once the theoretical model is built, it can be used to predict the corresponding surface geometry each time the operating conditions are known. Following a study on ceramics in 2009, Srinivasu [5] shows experimentally the influence of the angle of the cutting head on the depth and the width of the incision. He presented an analysis of the geometry of an incision by taking into account the kinematic parameters (impact angle and the feed rate of the jet) in AWJ machining of $\mathrm{SiC}$ ceramics, and added that the velocity of water and abrasive particles were assumed to follow the shape of Gaussian distribution.

Once the geometrical modeling is found, a prediction of the jet footprint can be made even in the case of changing process parameters. Axinte [13] used a finite 


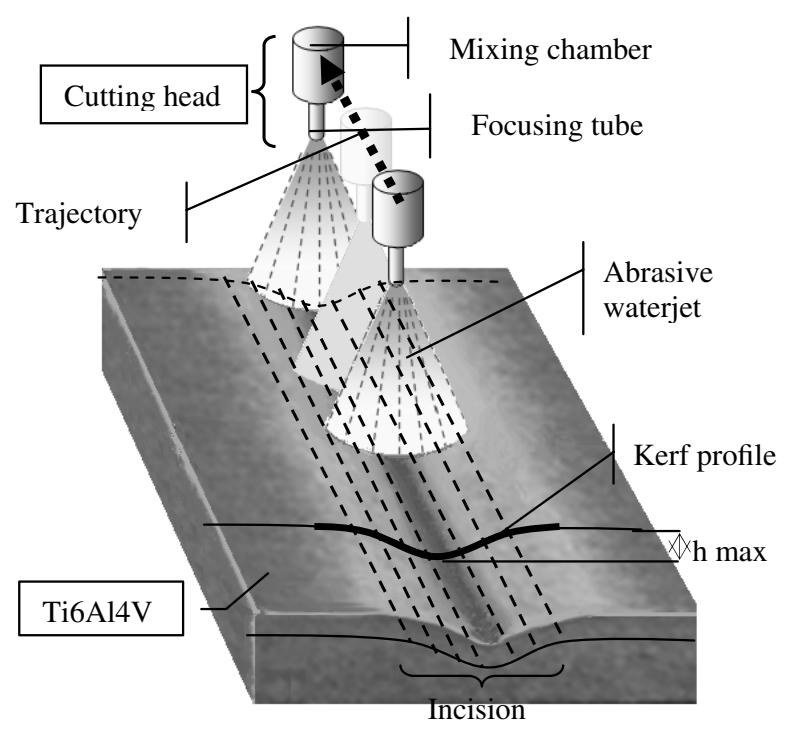

Fig. 4. Single path of the cutting head creating an incision on titanium alloys workpiece.

element model also based on Gaussian model explaining that abrasive particles follow a Gaussian distribution. In the same way, Alberdi [14-16] associated the kerf profile obtained after a rectilinear passage of the jet on the workpiece, to a Gaussian shape. These studies showed that the energy distribution of the jet follows a Gaussian distribution due to the good implementation of the Gaussian bell with incision's form found using experimental tests. In addition the Gaussian distribution results from the random distribution of abrasive particles during mixing with water particles and air. According to his study, the other advantage of using the Gaussian function (Eq. (1)) is to describe the profile of an incision with only two coefficients to be modeled using at first the maximum cutting depth factor and secondly the width factor:

$$
h=h_{\max } \mathrm{e}^{\frac{-b^{2}}{2 c^{2}}} \quad \text { where } \quad c=\frac{b_{0.5}}{2 \sqrt{(2)(\ln 2)}}
$$

In this expression, $h_{\max }$ is the incision's maximum depth and $b_{0.5}$ is the width at half of the maximum depth.

On the other hand, Cénac [1] presented an erosion model separating micro-cutting (abrasion mechanism) and fragile fracture (ploughing mechanism) based on a calculation of the kinetic energy of the abrasive particles made by Finnie [10] and affecting the workpiece. Erosion models for predicting depth of cut in AWJ require determining the velocity of the particles that involves measures in order to be calculated. The methods used to determine these velocities are difficult to implement and require a significant numerical processing. Thus, Cénac expressed the depth of the incision as a function of the pressure $P$, the traverse speed $f$, the flow of abrasive $D a$, the standoff distance $S O D$ and the diameter of the focusing tube $D c$. Cenac's model (Eq. (2)) is based only on the depth and does not consider the width of the incision.

$$
h=A 0 P^{A 1} D a^{A 2} f^{A 3} D c^{A 4} S O D^{A 5}
$$

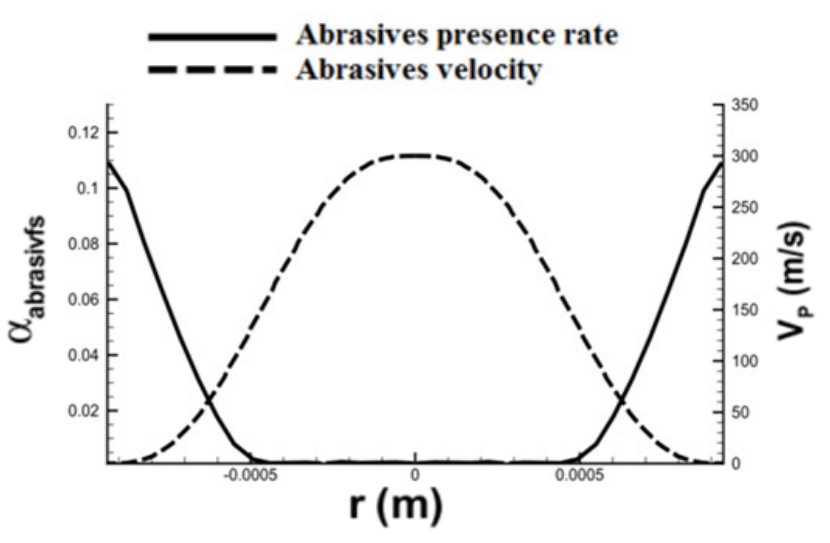

Fig. 5. Distribution of abrasive particles in the jet [19].

\subsection{Abrasive water jet phenomenology}

The present work, besides modeling the kerf profile of incisions, studies the physical phenomena when milling incisions in three-axis AWJM. It also focuses on the analysis of the phenomenology of the abrasive water jet flow and its precise influence on the result found on the workpiece. Several works explained the waterjet flowing and the dragging of abrasive particles in the jet $[17,18]$. Furthermore Zaki explained [19] that abrasive particles are accumulated on the borders of the mixing chamber (Fig. 4) while inflowing the focusing tube. He also showed that particles amassed in the conical area at the entrance of the focusing tube have a very low velocity while in the heart of the jet the velocity is very high and with very few abrasive particles (Fig. 5).

Kulekci [20] showed the path of abrasive particles flowing on the side of the focusing tube. Each particle enters the jet with a very low velocity. It is then accelerated by the jet on its way into the focusing tube. By hitting the tube sides this particle reaches a parallel trajectory to the jet axis.

\section{Modelling analysis of incision's profile}

The most used equation in AWJM $[5,14,15]$ to fit incision profile is the Gaussian model Equation (3):

$$
z(x)=a \mathrm{e}^{-\left(\frac{x-x_{0}}{b}\right)^{2}}
$$

where: $a=$ amplitude (maximum depth), $x_{0}=$ adjusting stance on $x$ axis and $b=$ width parameters.

The Gaussian model (Eq. (3)) and experimental incision profile are shown in Figure 6. A good correlation is seen in the higher part of the profile unlike the lower part where the Gaussian model does not well correlate with the experimental profile.

Some incisions, especially when machining with high SOD, showed some unconformity between Gaussian curves and experimental incision form particularly in the border zone of the kerf profile (Fig. 6). This observation 


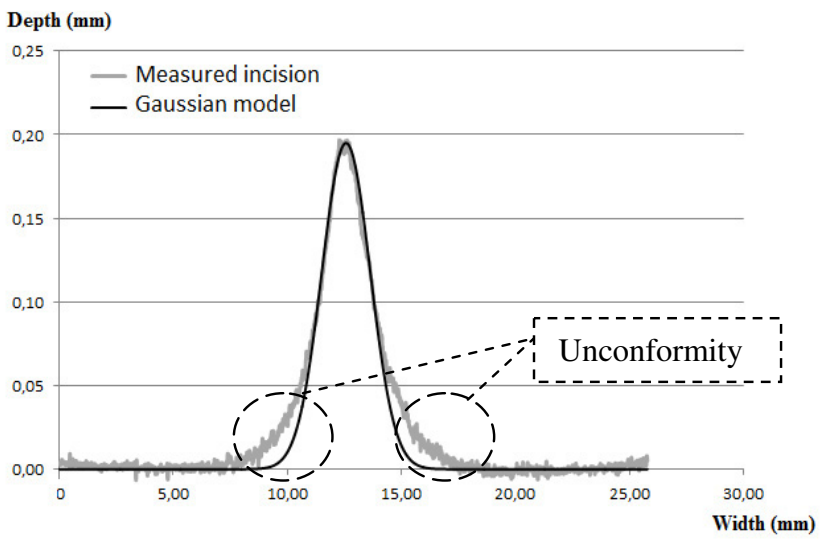

Fig. 6. Incision profile fitted with Gaussian model.
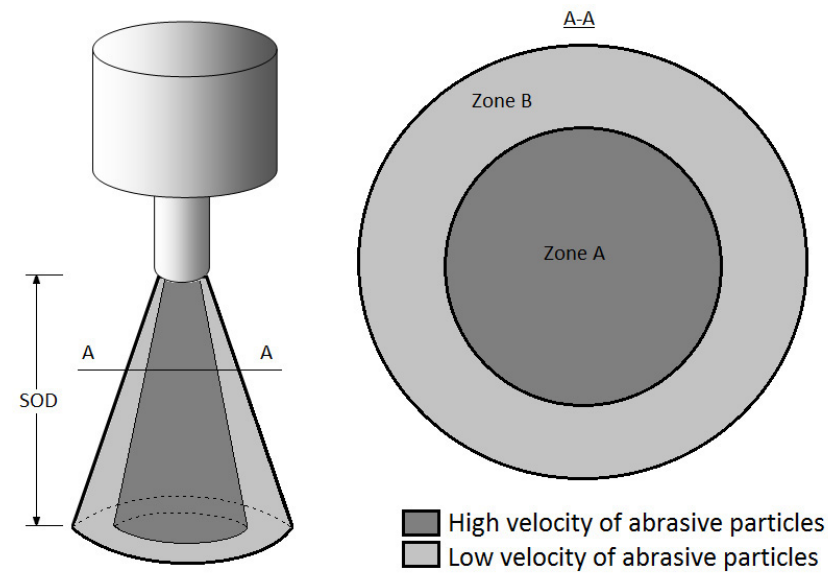

Fig. 7. Characterization of abrasive velocity in the jet.

leads to a deep study on the phenomenology of the jet and on the theoretical models in order to find the most suitable model for incision's profile obtained in AWJM of Ti6AlV.

Figure 6 shows that Gaussian model is not taking into account a distribution taking place especially in the border zone of the kerf profile. This remark leads to a belief that there are two main zones in the abrasive waterjet not flowing similarly [17]. Zone "A" is the central zone of the jet where the least quantity of abrasive particles is going at very high velocity (Fig. 7). And secondly the zone "B" where the biggest amount of abrasive particles is going at low velocity [19]. This theory leads to a deduction that each zone is having its proper Gaussian distribution.

The larger SOD is, the bigger the zone B will become comparing to the zone A. Therefore incision's profile obtained in that case will diverge from the normal Gaussian distribution and it will be more consistent to be represented by an extended Gaussian model. This means that a first normal Gaussian distribution characterizes the distribution of the first zone and a second Gaussian model characterizes the second one. This leads to a sum of the two Gaussian models that represents the entire distribution taking place in the abrasive water jet. Moreover, the smaller SOD is, the minor the zone B will become. Thereby the distribution of abrasive particles will tend to a Gaussian model. So the second Gaussian model representing the zone $\mathrm{B}$ will be negligible comparing to the entire jet and won't have a significant influence on the profile obtained on the workpiece. For small SODs the model fitting the measured profile of incision emerges to a normal Gaussian profile which joins the hypothesis of the consistence of the Gauss distribution found in literature $[6,14,21]$.

To improve Gaussian modeling, other mathematical functions exist to correlate with the bell's shape. Thereby, many were interesting to scoop out Figure 9:

\section{- Lorentz model:}

The Lorentz distribution is often used in statistics and has a bell's shape. By adding the depth parameter " $a$ " and the parameter " $x_{0}$ " adjusting the stance on the $x$ axis, the Lorentz equation becomes Equation (4):

$$
z(x)=a \frac{b^{2}}{2} \frac{1}{\frac{1}{2} b^{2}+\left(x-x_{0}\right)^{2}}
$$

$-a=$ amplitude (maximum depth);

$-x_{0}=$ adjusting stance on $x$ axis;

$-b=$ width parameter.

- Pearson model :

The Pearson model is usually used for peak detection in X-ray diffraction measurement. A simplified Pearson equation is:

$$
z(x)=a\left(1+\frac{K^{2}}{M}\left(x-x_{0}\right)^{2}\right)^{-M}
$$

where $a$ is the maximum depth, $K$ and $M$ are parameters that define width.

\section{- Gauss decomposed (GD) model:}

Gaussian distribution characterizes the best random distribution of particles in the jet, and is the finest model representing the physics of abrasive water jet. For this reason it was selected and improved using two parts corresponding to the two different distributions subsisting in the same jet (Fig. 8). Assuming that two similar phenomena exist but not flowing identically in the jet, the corresponding model to fit incision's form will be written as follows:

$$
z(x)=\frac{a}{2} \mathrm{e}^{-\left(\frac{x-x_{0}}{b_{1}}\right)^{2}}+\frac{a}{2} \mathrm{e}^{-\left(\frac{x-x_{0}}{b_{2}}\right)^{2}}
$$

$-a=$ amplitude (maximum depth);

$-x_{0}=$ adjusting stance on $x$ axis;

$-b_{1}$ and $b_{2}=$ width parameters relative to each distribution.

The amplitude coefficients multiplying Gaussian parts in this model were $a_{1}$ and $a_{2}$ respectively. Nevertheless several testings were used to define the most appropriate amplitude coefficient multiplying each Gaussian part of the 


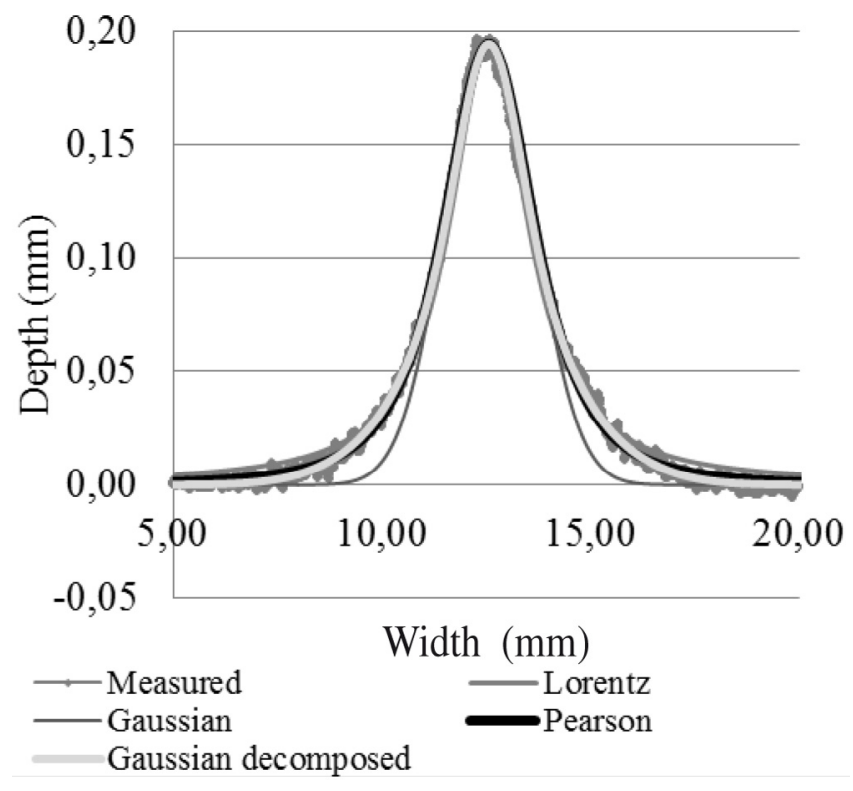

Fig. 8. Theoretical models fitting incision profile.



Fig. 9. Difference between measured value and theoretical value $=$ ri.

GD model. The difference, using the comparison with the minimum SSE (Sum Square Error), between amplitude coefficients was negligible. And the most suitable amplitude coefficient fitting the measured profiles in all trials of incisions was $a_{1}=a_{2}=Z_{\max } / 2$ which explains the use of $a / 2$ in the GD equation.

All models presented before are computed in Figure 8.

\section{Least square method for nonlinear equations}

The goal is to find the parameters value for the model which "best" fits the measured profile. It is used in this paper for the four nonlinear Equations (3)-(6).

A residual is defined as the difference between the actual value of the dependent variable and the value predicted by the model (Fig. 9):

$$
r i=z_{\text {measured }}-z_{\text {model }}
$$

Table 1. Sum Square Error (SSE) between measured and theoretical profiles for arbitrary incision.

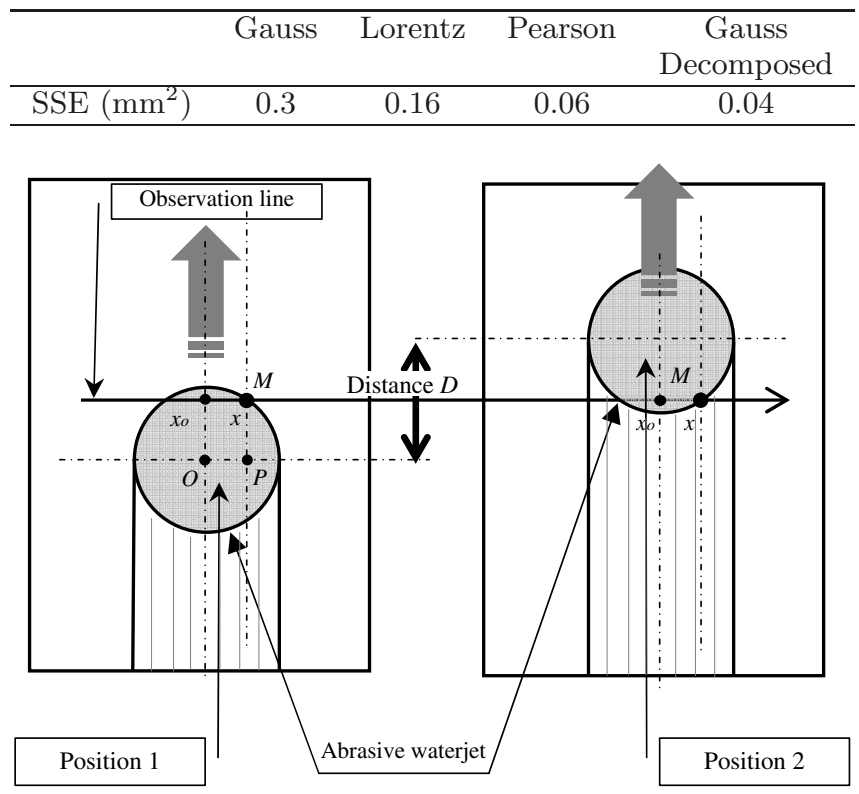

Fig. 10. Length exposure time for an arbitrary point $M$ on the incision's profile.

Consideration of the jet exposure time on the model of incisions:

The impact area of the water jet is being considered cylindrical and the incision is rectilinear. Each point $M$ on the workpiece (Fig. 10), located under the jet does not undergo the same exposure time. Let us consider an incision profile represented on an observation line shown in (Fig. 10). The jet reaches the point $M$ belonging to the incision profile and located at the abscissa $x$ as presented in position 1. Also, from the position 2, the point $M$ is no longer affected by the jet.

The distance $D$ (Fig. 10) characterizes the length covered by the jet in which it affects the point $M$ of the incision's profile. This distance depends on the $x$ coordinate considered on the observation line and is equal to:

$$
D=2 \times\|P M\|
$$

Figure 11 shows that the distance PM can be calculated as a function of the radius $R$ of the jet and thus the distance $D$ can be expressed:

$$
D=2 \times \sqrt{R^{2}-\left(x-x_{0}\right)^{2}} ; \quad \text { for } \quad x \in\left[x_{0}-R ; x_{0}+R\right]
$$

The distance $D$ is the image of the exposure time of the point $M$ of the jet. It should be taken into account in the expression of the incision's model which becomes:

$$
\begin{aligned}
z(x)=A & \left(\mathrm{e}^{-\left(\frac{x-x_{0}}{b_{1}}\right)^{2}}+\mathrm{e}^{-\left(\frac{x-x_{0}}{b_{2}}\right)^{2}}\right) 2 ; \\
& \times \sqrt{R^{2}-\left(x-x_{0}\right)^{2} / R} \text { with } A=a K .
\end{aligned}
$$

This model was implemented and the least squares method was used to determine the coefficients of the expression (10). Then for each incision studied, the sum 


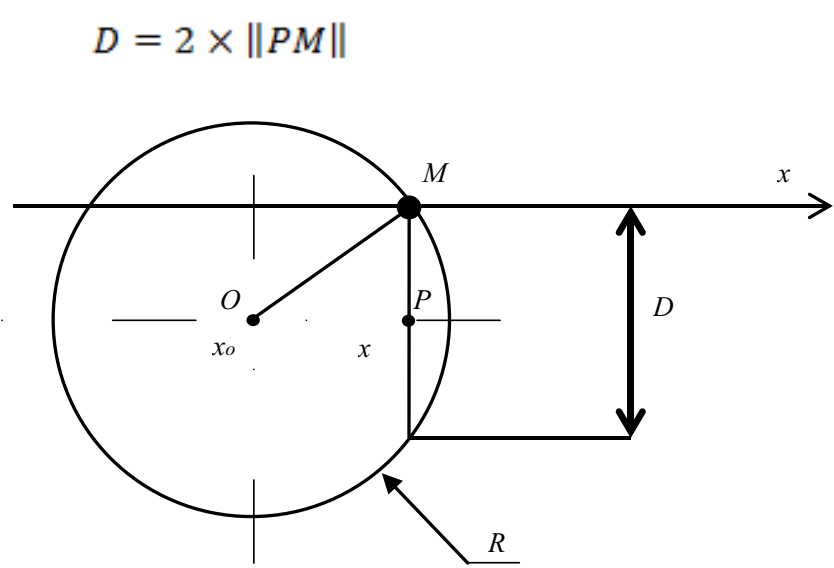

Fig. 11. The distance $D$ and the diameter of the jet.

square error corresponding to this model was compared to the one obtained by the Gauss Decomposed model (6).

\section{Conclusion on the use of this model}

The results showed that this model can only slightly and punctually improve the results of the prior "Gauss decomposed" model. The difference between the results is less than $5 \%$ and it shows that the energy distribution represented by the "Gauss decomposed" model is the largest contribution to the modeling of incisions. The exposure time does not have a significant effect in the case of straight incisions but may be important for those guided along curved trajectories.

\section{Experimental procedure}

To be sure that GD model has the best consistence with the measured incision profile, an extensive set of trials took place on a Flow Mach 3 AWJ machine. The experience presented in this sheet includes 27 incisions made on titanium alloys specimens with a constant pressure (1000 bars), with a silicon carbide 80 mesh abrasive granules, and an abrasive flow rate $\left(0.34 \mathrm{~kg} . \mathrm{min}^{-1}\right)$ involving various ranges of process parameters:

Traverse speed $f\left(\mathrm{~mm} \cdot \mathrm{min}^{-1}\right)(66-2182)$.

Standoff distance $s(\mathrm{~mm})(30 ; 80 ; 140)$.

Once incisions were made on titanium samples, several measures were done on Alicona optical 3D profilometer for high resolution $\left(10^{-5} \mathrm{~mm}\right.$ on $Z$ axis $)$ measurement (Fig. 12).

Each measurement is an acquisition on a surface of $16 \mathrm{~mm} \times 2 \mathrm{~mm}$ (Fig. 13).

Then a line is drawn on the measured surface giving the coordinates of 6000 points which is accurately enough to draw the measured incision's profile (Fig. 14).

The four theoretical models were applied to fit on each incision. The least square method for nonlinear equations was used and a comparison of the minimum of SSE was set.



Fig. 12. Measuring tool: Alicona 3D optical profilometer for high resolution measurement.

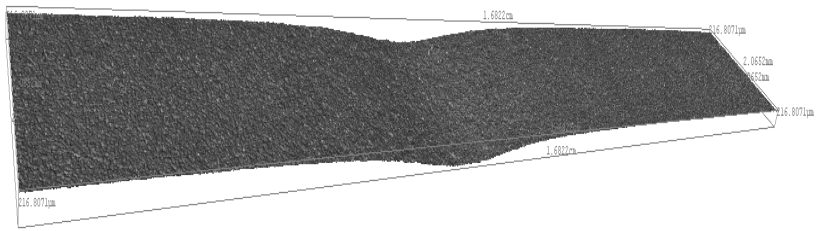

Fig. 13. Acquisition of the measured surface.

\section{Results and discussions}

In order to study the best model fitting the incision profile, models presented earlier were used and compared. The results show that 21 incisions were best fitted with Gauss decomposed (GD) model, 6 incisions with Pearson model and none with Gaussian or Lorentz model using SSE minimum as a comparison criterion. For 6 incisions, Pearson outweighs the GD model. The difference, between SSE values for Pearson and Gauss decomposed model, is insignificant (around $10^{-3} \mathrm{~mm}$ ). Thereby GD model shows the best correlation with the measured profiles for any value of SOD and traverse speed which makes it a new accurate model to fit kerf shapes in AWJM.

These outcomes are in harmony with the theory raised in Section 2 according to Zaki [19]. The profile obtained by a single jet path in AWJ machine is not only a Gaussian profile but a sum of two Gaussian profiles which are the two parts of the Gauss decomposed model corresponding to the two physical phenomena occurring in AWJ:

- The first part is the central one where are the highest velocity and the lowest quantity of abrasive particles.

- The second sub-jet is the lateral one where are the biggest amount of abrasive particles and the lowest velocity of the jet. 
T. Sultan et al.: Mechanics \& Industry 17, 403 (2016)



Fig. 14. The result of incision profile measured on Alicona.

Table 2. Milling process parameters and models comparison.

\begin{tabular}{|c|c|c|c|c|c|c|}
\hline \multicolumn{2}{|c|}{ Operating parameters } & \multicolumn{5}{|c|}{ SSE $\left(\mathrm{mm}^{2}\right)$} \\
\hline $\mathrm{SOD}(\mathrm{mm})$ & Traverse speed $\left(\mathrm{mm} \cdot \mathrm{min}^{-1}\right)$ & Gauss & Lorentz & Pearson $(\mathrm{P})$ & Gauss Decomposed (GD) & Best model \\
\hline 30 & 263 & 0.067 & 0.504 & 0.045 & 0.043 & GD \\
\hline 30 & 383 & 0.059 & 0.328 & 0.028 & 0.027 & GD \\
\hline 30 & 406 & 0.048 & 0.284 & 0.048 & 0.048 & $\mathrm{P}$ \\
\hline 30 & 581 & 0.055 & 0.081 & 0.055 & 0.034 & GD \\
\hline 30 & 724 & 0.032 & 0.100 & 0.029 & 0.029 & $\mathrm{P}$ \\
\hline 30 & 811 & 0.019 & 0.096 & 0.019 & 0.019 & $\mathrm{P}$ \\
\hline 30 & 1085 & 0.016 & 0.049 & 0.015 & 0.016 & $\mathrm{P}$ \\
\hline 30 & 1162 & 0.019 & 0.034 & 0.016 & 0.015 & GD \\
\hline 30 & 2182 & 0.015 & 0.022 & 0.015 & 0.014 & GD \\
\hline 80 & 110 & 0.351 & 1.337 & 0.190 & 0.187 & GD \\
\hline 80 & 164 & 0.196 & 0.361 & 0.038 & 0.027 & GD \\
\hline 80 & 175 & 0.111 & 0.352 & 0.093 & 0.095 & $\mathrm{P}$ \\
\hline 80 & 252 & 0.358 & 0.214 & 0.062 & 0.064 & $\mathrm{P}$ \\
\hline 80 & 307 & 0.065 & 0.155 & 0.037 & 0.037 & GD \\
\hline 80 & 351 & 0.052 & 0.125 & 0.042 & 0.04 & GD \\
\hline 80 & 460 & 0.100 & 0.210 & 0.088 & 0.085 & GD \\
\hline 80 & 504 & 0.039 & 0.062 & 0.023 & 0.02 & GD \\
\hline 80 & 932 & 0.025 & 0.055 & 0.025 & 0.024 & GD \\
\hline 140 & 66 & 0.952 & 0.416 & 0.410 & 0.338 & GD \\
\hline 140 & 98 & 0.244 & 0.389 & 0.128 & 0.12 & GD \\
\hline 140 & 104 & 0.317 & 0.350 & 0.051 & 0.037 & GD \\
\hline 140 & 153 & 0.137 & 0.210 & 0.045 & 0.03 & GD \\
\hline 140 & 186 & 0.141 & 0.146 & 0.064 & 0.06 & GD \\
\hline 140 & 209 & 0.068 & 0.096 & 0.068 & 0.058 & GD \\
\hline 140 & 285 & 0.056 & 0.093 & 0.041 & 0.041 & GD \\
\hline 140 & 307 & 0.061 & 0.065 & 0.032 & 0.031 & GD \\
\hline 140 & 581 & 0.036 & 0.059 & 0.036 & 0.035 & GD \\
\hline
\end{tabular}




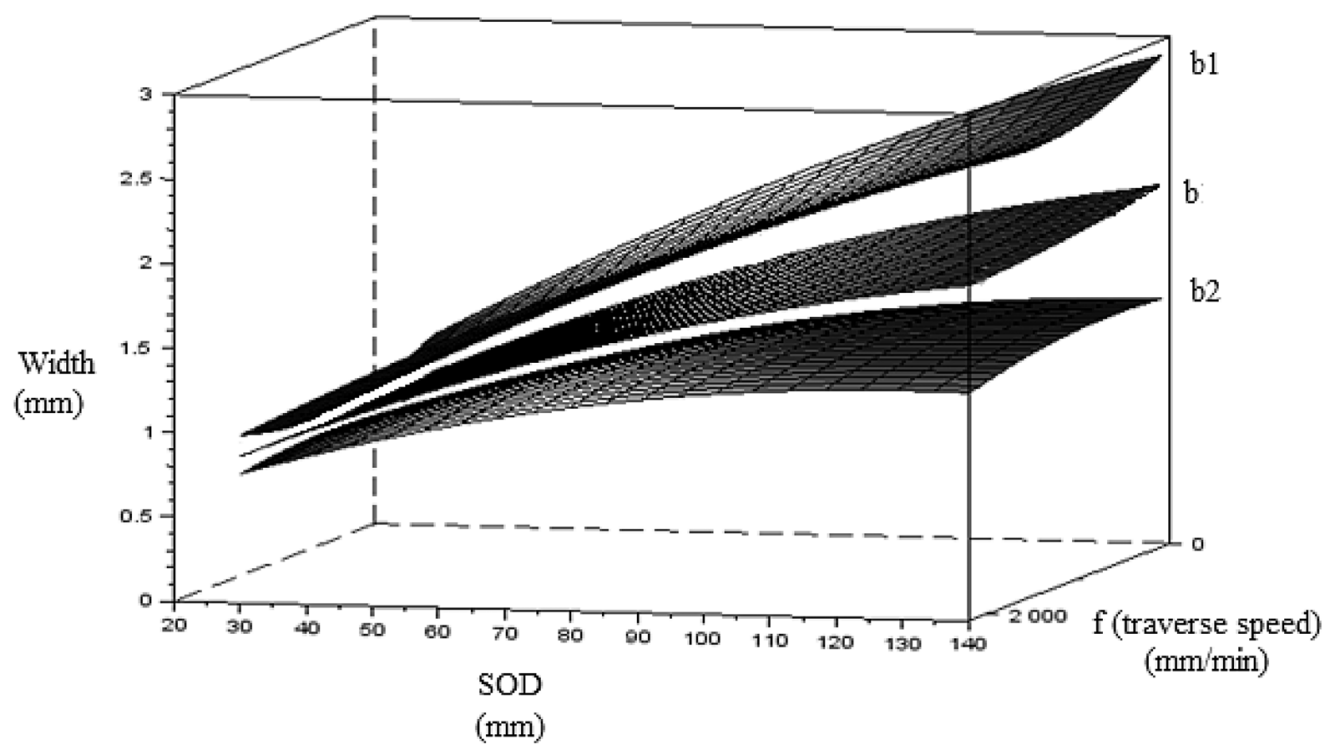

Fig. 15. Relation between width parameter for Gaussian and Gauss decomposed models.

These results give clear evidence that the Gauss decomposed model is the most suitable theoretical model in AWJM.

The proposed model integrates three variables ( $a$ for the depth, $b_{1}$ and $b_{2}$ for the width) instead of two parameters for the Gaussian model ( $a$ for the depth and $b$ for the width). The coefficient $b$ representing the width of the Gaussian model is the mean of the two coefficients $b_{1}$ and $b_{2}$. Figure 15 shows calculated width values $b, b_{1}$ and $b_{2}$ for the 27 incisions made on titanium alloys.

\subsection{Comments in Figure 15}

- When SOD is small, calculated values $b_{1}$ and $b_{2}$ tend to the calculated value $b$. This means that the Gauss decomposed model converges to a simple Gaussian model.

- Traverse speed seems to have a negligible effect on incision's width unlike SOD which has a remarkable influence.

\section{Conclusion and further work}

This paper presents a study based on theoretical models fitting the footprint done by a single path of the jet on titanium workpiece. On the first hand, most of works describe this footprint resulting of a single toolpath in AWJ as a Gaussian profile. On the other hand, some works show that abrasive particles are accumulated on the borders of the mixing chamber. Consequently incisions obtained while milling in AWJ can't be fitted with a simple Gaussian model. This hypothesis drives the analytical study on theoretical models to search other formulas of bell shapes. The theoretical study used in this sheet gives clear evidence that the Gaussian profile is not the most suitable theoretical model in AWJ milling. Results show that the Gauss decomposed model is the most efficient when using a large interval of traverse speed and SOD. This new model takes into account the particularity of the jet. In small SOD the second part of the Gauss decomposed model characterizing the zone of low velocity will be negligible and the final Gauss decomposed model will tend to a normal Gaussian model. And in the other hand, with high SOD the second part will have a greater effect and the model fitting the footprint of incision is the two Gaussian model characterizing the two zones of the jet.

Once the most accurate theoretical model for incisions is found, the predictive equation of depth and width of cut for titanium alloys can be set. Further studies will include this predictive equation to define predictive models for incisions and pockets in abrasive waterjet milling of titanium alloys.

\section{References}

[1] F. Cenac, Étude de l'usinage non débouchant par jet d'eau abrasif des composites, Ph.D thesis, University Paul Sabatier, 2011

[2] L. Crouzeix, F. Collombet, Y. Grunevald, Step repaired coupons involving Abrasive Water Jet machining, in: JNC 17, Poitiers, 2011, pp. 1-10

[3] G. Fowler, Abrasive Water-jet - Controlled Depth Milling Titanium Alloys, Ph.D. thesis, University of Nottingham, 2003

[4] D.S. Srinivasu, D. Axinte, An analytical model for top width of jet footprint in abrasive waterjet milling: a case study on SiC ceramics, in: Proc. Inst. Mech. Eng., 2011, pp. 319-335 
[5] D.S. Srinivasu, D.A. Axinte, P.H. Shipway, J. Folkes, Influence of kinematic operating parameters on kerf geometry in abrasive waterjet machining of silicon carbide ceramics, Int. J. Mach. Tools Manuf. 49 (2009) 1077-1088

[6] N. Zuckerman, N. Lior, Jet Impingement Heat Transfer: Physics, Correlations, and Numerical Modeling, Adv. Heat Transf. 39 (2006) 565-631

[7] M.C. Kong, D. Axinte, W. Voice, Aspects of material removal mechanism in plain waterjet milling on gamma titanium aluminide, J. Mater. Process. Technol. 210 (2010) $573-584$

[8] M.C. Kong, D. Axinte, W. Voice, Challenges in using waterjet machining of NiTi shape memory alloys: An analysis of controlled-depth milling, J. Mater. Process. Technol. 211 (2011) 959-971

[9] J. Wang, Abrasive Waterjet Machining of Polymer Matrix Composites - Cutting Performance, Erosive Process and Predictive Models, Int. J. Adv. Manuf. Technol. 15 (1999) 757-768

[10] I. Finnie, Erosion of surface by solid particles, Shell Dev. Co. 1960

[11] H.T. Zhu, C.Z. Huang, J. Wang, Q.L. Li, C.L. Che, Experimental study on abrasive waterjet polishing for hard-brittle materials, Int. J. Mach. Tools Manuf. 49 (2009) 569-578

[12] K. Maniadaki, T. Kestis, N. Bilalis, A. Antoniadis, A finite element-based model for pure waterjet process simulation, Int. J. Adv. Manuf. Technol. 31 (2006) 933-940

[13] S. Anwar, D.A. Axinte, A.A. Becker, Finite element modelling of overlapping abrasive waterjet milled footprints, Wear (2013)
[14] A. Alberdi, A. Rivero, L.N. López de Lacalle, I. Etxeberria, A. Suárez, Effect of process parameter on the kerf geometry in abrasive water jet milling, Int. J. Adv. Manuf. Technol. 51 (2010) 467-480

[15] A. Carrascal, A. Alberdi, F. Fatronik-Tecnalia, P. Mikeletegi, P. Tecnológico, Evolutionary Industrial Physical Model Generation, 2010, pp. 327-334

[16] A. Alberdi, A. Rivero, L.N. López de Lacalle, Experimental Study of the Slot Overlapping and Tool Path Variation Effect in Abrasive Waterjet Milling, J. Manuf. Sci. Eng. 133 (2011) 034502

[17] A. Cornier, Developpement d'un modèle d'enlèvement de matière par granulation utilisant le jet d'eau haute pression: application au démantelement de pneumatiques, Ph.D. Thesis, École Nationale Superieure d'Arts et Metiers, 2004

[18] S. Ferrendier, Influence de l'Evolution Granulométrique des Abrasifs sur l'Enlèvement de Matière lors de la découpe par JEA, Ph.D. thesis, École Nationale Superieure d'Arts et Metiers, 2001

[19] M. Zaki, Modélisation et simulation numérique du procédé de perçage non débouchant par jet d'eau abrasif, Ph.D. thesis, École Nationale Superieure d'Arts et Metiers, 2009

[20] M.K. Kulekci, Processes and apparatus developments in industrial waterjet applications, Int. J. Mach. Tools Manuf. 42 1297-1306. 42 (2002) 1297-1306

[21] D.A. Axinte, D.S. Srinivasu, J. Billingham, M. Cooper, Geometrical modelling of abrasive waterjet footprints: A study for $90^{\circ}$ jet impact angle, CIRP Ann. - Manuf. Technol. 59 (2010) 341-346 